\title{
Survival and Prognostic Factors in Patients with Glioblastoma Multiforme Receiving Radiation and Temozolomide - A Retrospective Study
}

\author{
Bindu S M' ${ }^{1}$ P L Rema ${ }^{2}$, Praveen Jacob Ninan ${ }^{3}$ \\ ${ }^{1}$ Assistant Professor of Radiotherapy, ${ }^{2}$ Professor of Radiotherapy, ${ }^{3}$ Associate Professor of Radiotherapy; \\ Govt T D Medical College, Vandanam, Alappuzha, Kerala, India
}

Corresponding Author: Praveen Jacob Ninan

\begin{abstract}
Introduction: Glioblastoma multiforme (GBM) is the most common primary brain tumor in adults. The standard treatment is surgical excision, followed by concomitant chemoradiation and adjuvant treatment with temozolomide. The standard radiation dose is 60Gy/ 30 fractions 2 Gy per fraction. Temozolomide is an alkylating agent and dosage is $75 \mathrm{mg} / \mathrm{m} 2$ concurrent with radiation and $150-200 \mathrm{mg} / \mathrm{m} 2$ as adjuvant treatment. This study aims at studying the median survival and the various prognostic factors.
\end{abstract}

Materials and Methods: This is a retrospective study conducted in Government Medical College, Alappuzha, of 44 patients with GBM from March 2015-2017, who underwent surgery followed by chemoradiation with temozolomide and six cycles of adjuvant temozolomide.

Result: Out of 44 patients, 26 were males and 18 females. There were $28(64 \%)$ patients with an ECOG performance status (PS) of $0-1$ and had a median survival (MS) of 21months and $16(36 \%)$ patients with PS of 2 who had a MS of 10 months ( $p$ value <0.001). In this study $30(68 \%)$ patients had complete or near complete excision and had a MS of 20 months and 14 (32\%) patients had only a partial excision who had a MS of 10 months(p value $<0.001$ ). There were $25(57 \%)$ patients below 50 years who had a MS of 20 months and 19(43\%) patients above 50 years who had a MS of 12 months ( $\mathrm{p}$ value $<0.01)$.

Conclusion: The prognostic factors detected were age, performance status and extent of resection. The triple modality of treatment of concurrent chemoradiation with temozolomide followed by adjuvant temozolomide continues to be the standard treatment.

Keywords: Glioblastoma multiforme,

Temozolomide, chemoradiation

\section{INTRODUCTION}

Glioblastoma multiforme is a primary brain tumor which accounts for $2 \%$ of all malignancy, occurring in adults ${ }^{(1)}$. Glioblastoma multiforme is a grade IV glioma according to WHO 2016 classification, with incidence higher in males (2) and the name is coined by Cushings in the nineteenth century ${ }^{(3)}$. It is usually seen at older age group with a median age of 64 years and uncommon in children ${ }^{(2)}$. In India brain tumors are ranked the tenth most common type of tumor by the International Association of Cancer (IAC) and accounts for 28000 new cases each year (4). Glioblastoma is most commonly situated in the supratentorial region (frontal, temporal, parietal and occipital) with the highest incidence in the frontal lobe, followed by temporal and parietal lobes ${ }^{(3)}$. GBM presents in $13 \%$ patients as multifocal that is including more than two lesions including leptomeningeal dissemination ${ }^{(5)}$. GBM is rarely located in the cerebellum and extremely rare in the spinal cord ${ }^{(6)}$. Ionizing radiation is one of the few known risk factors of GBM and electromagnetic fields, formaldehyde and nonionising radiations from cell phones have not being proven to lead to $\mathrm{GBM}^{(7)}$. 
Clinical presentation of the disease depends on the site and size of the tumor and the anatomical structures involved and includes headache, seizures, dizziness, loss of consciousness, speech or visual disturbances, weakness and confusion ${ }^{(8)}$. Seizure is the presenting symptom in 30$50 \%$ of patients having high grade glioma (8). The radiological investigations used are CT scan and MRI scan with gadolinium as contrast agent. Glioblastoma multiforme (GBM) enhances and shows a dense ring of enhancement around an irregularly shaped mass with hypointense centre of necrosis. Microscopic spread of disease extends beyond the visualized signal abnormality in Gadolinium enhanced MRI ${ }^{(5)}$. According to WHO classification necrosis in the tumor is considered to be the hallmark of GBM ${ }^{(5)}$.

Treatment of newly diagnosed GBM includes surgery, radiation and chemotherapy. Extensive and complete surgical resection of GBM is difficult and therefore comes the importance of adjuvant treatment via radiation and chemotherapy. Postop radiation (RT) alone was standard treatment until 2005. External beam radiation with concomitant chemotherapy using temozolomide (TMZ) is found to be more effective than radiation alone. ${ }^{(9)}$, Patients who received concurrent chemoradiation had a median survival of 14.6 months versus 12.1 months with RT alone ${ }^{(9)}$. Temozolomide (TMZ) is an orally bioavailable alkylating agent which is able to penetrate the blood brain barrier ${ }^{(10)}$. Temozolomide functions by addition of methyl group (methylation) of guanine at $\mathrm{N} 7$ and $\mathrm{O} 6$ sites and of adenine at the O3 site thereby modifying the DNA and RNA (10). TMZ is typically given at a dose of 75 $\mathrm{mg} / \mathrm{m} 2$ daily for 6 weeks concurrent with radiation and as adjuvant chemotherapy TMZ is given as $150-200 \mathrm{mg} / \mathrm{m} 2$ five days every 28 days ${ }^{(11)}$. Current standard of care in radiation treatment in GBM is 6000cGy in 30 fractions, 2 Gy per fraction five days a week with concurrent temozolomide ${ }^{(11)}$. In spite of multimodality of treatment by way of surgery, radiation and chemotherapy the median survival is only 14.6 months ${ }^{(12)}$. Very few studies have been conducted in Indian population regarding use of triple modality of treatment in GBM. So a retrospective data based study of the median survival with this treatment and the various prognostic factors was conducted in our hospital.

\section{MATERIALS AND METHOD}

This is a single Institution data based retrospective study conducted in the Department of Radiotherapy, Government Medical College, Alappuzha, Kerala. The study aims at detecting the median survival in months and prognostic factors affecting the survival of the patients who have received the current standard treatment protocol of postoperative concurrent chemoradiation with temozolomide, followed by adjuvant temozolomide for six months. After getting ethic clearance, all patients with GBM registered in the Radiotherapy Department during the period March 2015 to March 2017 for two years were selected in the study having the following characteristics,

\section{INCLUSION CRITERIA}

1. Patients with biopsy proven GBM

2. ECOG performance scale of 0 to 2

3. GBM patients who received the standard treatment of postop concurrent chemoradiation with temozolomide followed by adjuvant temozolomide for 6 months.

\section{EXCLUSION CRITERIA}

1. GBM patients with spinal cord involvement

The Case registers of all such patients were retrieved from the Cancer Registry and details collected regarding the patient characteristics like age, gender and sex, date of surgery, clinical presentation, tumor characteristics like size and site of the tumor, details of treatment and follow up data for two years. All patients had radiation treatment delivered using Linear Accelerator Varian Clinac IX with 
Megavoltage beams with appropriate photon energy.

\section{STATISTICAL ANALYSIS}

The data collected from the medical records were entered into MS Excel sheet and were analyzed with SPSS software version 18. The categorical variables are summarized using frequency and proportions, and quantitative variables like survival time is summarized using median and interval estimate is shown as 95\% confidence interval. To assess the difference in survival between various categories (excision, age and performance status), survival analysis was done using KaplanMeier method. The survival curves were tested using log rank (Mantel Cox) test and a $\mathrm{p}$ value of $<0.05$ is taken as statistically significant.

\section{RESULTS}

We have analyzed retrospectively the case records of 44 patients with histological diagnosis of GBM and who have received postoperative radiation with concurrent temozolomide during the period between March 2015 and 2017. The below are the data collected from the Cancer Registry files regarding patient characteristics like age, sex, ECOG performance scale, tumor characteristics like size and site of the tumor and the anatomical location, treatment details regarding surgery, radiation and chemotherapy, clinical presentation of the patients and follow up details.

\section{Patient Characteristics}

A total of 44 patients were studied. The age of the patients ranged from 32 years to 68 years. We have divided patients into two age groups that is < 50years and >50 years. There were 25 patients who were $<50$ years and 19 patients who were $>50$ years of age. Out of 44 patients, 26 patients were males and 18 were females. ECOG Performance scale (PS) (table 1) of $0-1$ was seen in 28 patients and the rest 16 patients had a PS of 2. Majority of our patients that is $68 \%$ had a good performance status. The completeness of surgery was considered under two headings i.e. those with complete resection (CR) and those with partial resection (PR). When $90 \%$ or more of the tumor is resected as studied from the postoperative MRI scan the term complete resection (CR) was used and anything less than $\mathrm{CR}$ was considered a PR. Complete resection was done in 30(68\%) patients and partial resection was done in $14(32 \%)$ patients.

Table 1: Eastern Cooperative Oncology Group (ECOG) Performance scale(13)

\begin{tabular}{|l|l|}
\hline GRADE & ECOG PERFORMANCE STATUS \\
\hline 0 & Fully active, able to carry out all pre-disease performance without restriction \\
\hline 1 & $\begin{array}{l}\text { Restricted in physically strenuous activity but ambulatory and able to carry out work of a light or sedentary nature eg; light } \\
\text { house work or office work }\end{array}$ \\
\hline 2 & Ambulatory and capable of all self care but unable to carry out any work activities up and about $>50 \%$ of waking hours \\
\hline 3 & Capable of only limited self care, confined to bed or chair $>50 \%$ of waking hours \\
\hline 4 & Completely disabled cannot carry on self care totally confined to bed or chair \\
\hline 5 & Dead \\
\hline
\end{tabular}

\section{Tumor Characteristics}

Tumor details were obtained from MRI scans which were taken both preop and postop. There were a total of 28 patients with right sided tumors, 14 in the left side and 2 in the midline. All patients had a histological proof of GBM. Most common presentation of the tumor was in the frontal lobe followed by parietal lobe. Patient and tumor characteristics are enlisted in Table 2.

\section{Clinical Presentation}

Patients presented with headache, nausea, vomiting, visual symptoms, weakness and convulsions. The most common presenting complaint was headache accounting for $92 \%$ followed by vomiting (56\%).

\section{Treatment Details}

Out of 44 patients only 30 had a complete or near complete excision of the 
Bindu S M et.al. Survival and prognostic factors in patients with glioblastoma multiforme receiving radiation and temozolomide - a retrospective study.

tumor and the rest 14 patients either had a partial resection or biopsy. All patients selected in our study had received radical radiation with $60 \mathrm{~Gy} / 30$ fractions with concurrent temozolomide followed by adjuvant temozolomide for six months .All patients received radiation in Linear Accelerator after CT simulation. Radiation was planned with 3DCRT or IMRT technique. Radiation dose given was 60 Gy/30 fractions once daily 5 days a week for 5 weeks with concurrent temozolomide $75 \mathrm{mg} / \mathrm{m} 2$ daily for 42 days till completion of radiation .Dose of temozolomide as adjuvant treatment was $150-200 \mathrm{mg} / \mathrm{m} 2$ day 1to day5 every 28 days for six months. Routine complete blood count, renal function tests and electrolytes were tested before giving chemotherapy.

\section{Follow up details}

After completion of treatment, patients were followed up every month for six months and every two months for the next 2 years. Follow up was done clinically, with blood investigations and radiological investigations by way of MRI scan. Table 2 and 3 show the patient and tumor characteristics.

Table 2: Patient characteristics

\begin{tabular}{|l|l|}
\hline Table 2: Patient characteristics \\
\hline Male & \\
\hline Female & $26(60 \%)$ \\
\hline ECOG Performance status & $18(40 \%)$ \\
\hline $0-1$ & \\
\hline 2 & $28(64 \%)$ \\
\hline Age & $16(36 \%)$ \\
\hline $30-40 \mathrm{yrs}$ & \\
\hline $40-50 \mathrm{yrs}$ & $7(16 \%)$ \\
\hline $50-60 \mathrm{yrs}$ & $18(41 \%)$ \\
\hline $60-70 \mathrm{yrs}$ & $14(32 \%)$ \\
\hline
\end{tabular}

Table 3: Tumor characteristics

\begin{tabular}{|l|l|}
\hline Tumor location & \\
\hline Right side & $28(64 \%)$ \\
\hline Left side & $14(32 \%)$ \\
\hline Midline & $2(04 \%)$ \\
\hline Age & \\
\hline$>50$ years & $19(43 \%)$ \\
\hline$<50$ years & $25(57 \%)$ \\
\hline Resection extent & \\
\hline Complete resection & $30(68 \%)$ \\
\hline Partial resection & $14(32 \%)$ \\
\hline
\end{tabular}

In our study the male patients formed the majority $(60 \%)$. There were 24 male patients and 16 female patients. The age of the patients ranged from 32 years to 68 years. For convenience of studying the results, we divided the patients into two groups $<50$ years and $>50$ years. More patients were in the $<50$ years group that is $25(57 \%)$ and this is in contrast with the study population of other standard studies where majority of patients were $>50$ years old. Performance status was assessed with the ECOG scale (table1) and a score of 0-1 was seen in $28(64 \%)$ patients and a score of 2 was seen in $16(36 \%)$ patients. Complete resection or near complete resection was done in $30(68 \%)$ patients and only partial resection was done in $14(32 \%)$ patients. The extent of resection was assessed radiologically and from the operative notes of the neurosurgeon. All patients had preop and postop MRI scans as these tests are done free of cost for financially poor patients in our hospital.

Table 4: Distribution of subjects based on age, performance status and excision status

\begin{tabular}{|l|l|l|l|}
\hline Variables & Categories & Frequency & Percent \\
\hline \multirow{2}{*}{ Age in years } & $<50$ yrs & 25 & 56.8 \\
\cline { 2 - 4 } & $>50$ yrs & 19 & 43.2 \\
\hline \multirow{2}{*}{ Performance status } & $0-1$ & 28 & 63.6 \\
\cline { 2 - 4 } & 2 & 16 & 36.4 \\
\hline \multirow{2}{*}{ Excision } & Complete & 30 & 68.2 \\
\cline { 2 - 4 } & Partial & 14 & 31.8 \\
\hline
\end{tabular}

Table 5: Comparison of median survival time of between different various categories $(n=44)$

\begin{tabular}{|l|l|l|l|}
\hline Variables & Subcategory & Median survival time (months) & $\mathbf{9 5 \%}$ Confidence Interval (months) \\
\hline \multirow{2}{*}{ Excision category } & Complete & 20 & $18.81-21.19$ \\
\cline { 2 - 4 } & Partial & 10 & $6.33-13.66$ \\
\hline \multirow{2}{*}{ Age in years } & $<50 \mathrm{yrs}$ & 20 & $18.91-21.08$ \\
\cline { 2 - 4 } & $>50 \mathrm{yrs}$ & 12 & $9.94-14.06$ \\
\hline \multirow{2}{*}{ Performance status } & $0-1$ & 21 & $19.89-22.10$ \\
\cline { 2 - 4 } & 2 & 10 & $6.08-13.92$ \\
\hline
\end{tabular}


Bindu S M et.al. Survival and prognostic factors in patients with glioblastoma multiforme receiving radiation and temozolomide - a retrospective study.

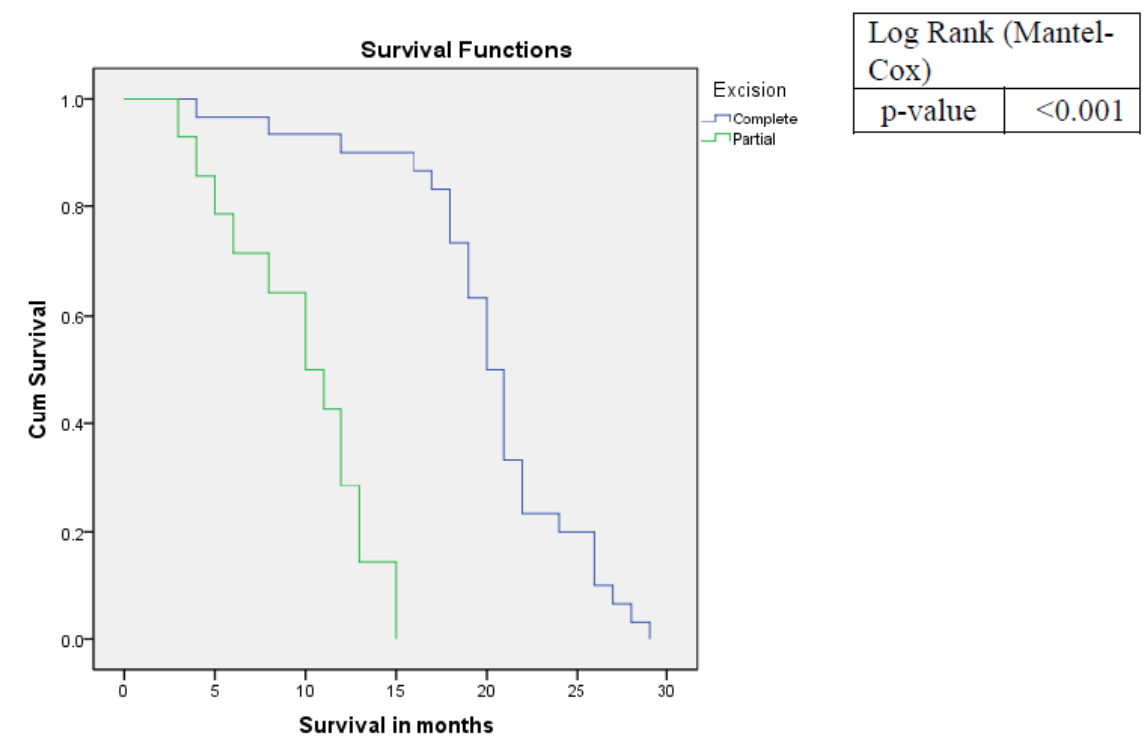

GRAPH I: Comparison of Median Survival Time of excision categories (complete and partial)

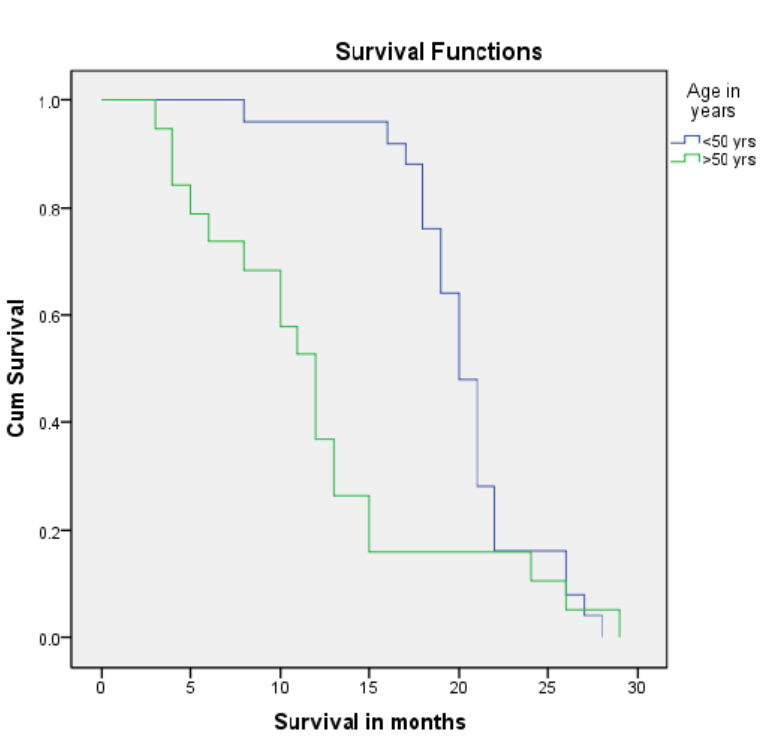

\begin{tabular}{|l|l|}
\hline \multicolumn{2}{|l|}{ Log Rank (Mantel- } \\
Cox) \\
\hline p-value & 0.01 \\
\hline
\end{tabular}

GRAPH II: Comparison of Median Survival Time between different age categories

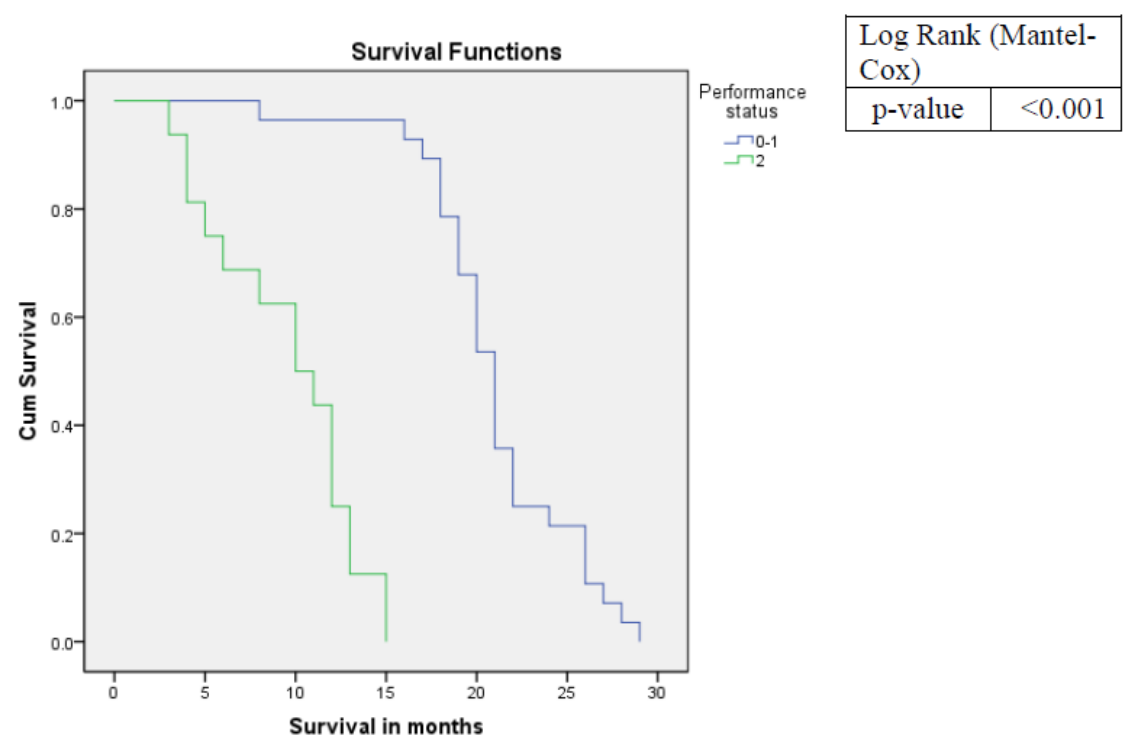

GRAPH III: Comparison of Median Survival Time between performance status categories 
Complete resection (CR) was done in $30(68 \%)$ patients and had a median survival of 20 months and $14(32 \%)$ patients had only a partial resection (PR) of tumor and had a median survival of 10 months (Graph I). Here there is a statistically significant advantage in survival of patients with complete excision of the tumor ( $p$ value is $<0.001$ ).

The age group of $<50$ years had a median survival of 20 months which constituted $25(57 \%)$ of patients and patients $>50$ years which constituted 19 (43\%) patients had a median survival of 12 months (Graph II). In this study three patients with age group $>50$ years had a good survival of 24, 26 and 26 months each and the lowest survival recorded in this group was 3 months. Even though the number of patients was small there was a trend towards improvement in median survival in lesser age group patients ( $\mathrm{p}$ value $<0.01$ ) which is statistically significant.

In patients with ECOG performance status (PS) of 0-1 the median survival was 21 months and those with performance status of 2 had a median survival of 10 months, 64\% of our patients had a PS of 0-1 and $36 \%$ had a PS of 2(Graph III). Here also patients with good PS had a better survival which is statistically significant ( $\mathrm{p}$ value $<0.001$ ). Majority of our patients had a better performance status. Patients with poorer performance status presented with convulsions and loss of consciousness and also had more neurological deficits. So patients who had convulsions at presentation bore a poor prognosis.

\section{DISCUSSION}

Glioblastoma multiforme is the most common primary brain tumor with grave prognosis having a median survival of 5 months without any treatment ${ }^{(14)}$. The classical treatment of GBM is surgery followed by radiation with concomitant temozolomide and adjuvant temozolomide. Supportive care forms an integral part of GBM treatment as patients may have neurological symptoms which may be progressive and will have to be managed with, antiepileptics and drugs to reduce intracranial pressure ${ }^{(8)}$. Antiepileptics are used only in patients with epilepsy, and not prescribed to patients without epilepsy ${ }^{(8)}$

In this study the age of the patients ranged from 32 years to 68 years. There were 26 male patients and 18 female patients and the male female ratio of the patients was 3:2. This is in accordance with the incidence of GBM in other studies which have shown that males are $60 \%$ more likely to develop $\mathrm{GBM}^{(15)}$. According to the statistics by Straube et al the age group affected by GBM is $45-70$ years and the median age is 64 years ${ }^{(15)}$. In our study the patients were divided into < 50years and $>50 y e a r s$ of age and number of patients $<50$ years was $25(57 \%)$ and those $>50$ years was 19(43\%). In the standard studies the age group with> 50 years had the maximum number of patients which was in contrast with our study population. The median survival of $<50$ years was 20 months as against 12 months in the $>50$ years age (p value $<0.01$ ). Even though the size of the study is small the median survival is statistically significant with a definite advantage in the $<50$ years age group. In the Ghosh study the number of patients were more in the $<50$ years age group and the median survival was maximum in the 31-40 years of age group, ${ }^{(16)}$. The probable reason for GBM to occur in lower age group in India was given as a low life expectancy of population in developing country like India (16). The Medical Research Council divided the GBM patients into age groups $<45$ years, 45-59 years and >59 years with median survival of 12 months, 9 months and $<5$ months respectively (16). Recursive Partitioning Analysis (RPA) model is a set of classes developed by Curran et al in which the most important split was by age and has taken 50 years the break point ${ }^{(16)}$. Age and Performance Status were detected to be the most important prognostic factors in GBM by this novel study by Curran et al (17). The median survival of patients with age $<50$ years with a good performance 
status was 18 months and those with age > 50 years and poor performance status was 5 months ${ }^{(17)}$. Studies have shown that patients with increased age have a decreased survival. Elderly patients or patients with poor performance status have poor prognosis this may be because of their other co-morbidities and also the inability to tolerate the standard trimodality treatment. (18)

The performance statuses of the patients were analyzed according to ECOG Performance scale (table 1). In our study $28(64 \%)$ patients had a performance status of $0-1$ and the rest 16 (36\%) had a performance status of 2 . We obtained a median survival of 21 months in better performance patients and median survival of 10 months for patients with performance status of 2 ( $\mathrm{p}$ value $<0.001$ ) which is statistically significant. A study for detecting the prognostic factors in GBM by Lutterbach et al detected a median survival of 8.8 months in patients with performance status (Karnofsky Performance Scale) KPS $>70$ and 6.7 months in KPS $<70^{(19)}$. In our study also we have detected an increased median survival in good performance patients when compared with poor performance patients. So better the performance status better chance of improved survival. The retrospective study of prognostic factors by Narendra Kumar et al has shown a median survival of 6.33 months with poor KPS status and 7.97 with better KPS status. ${ }^{(20)}$. Lacroix et al in a retrospective analysis showed that median survival of 8.8 months was present with KPS $<70$ and median survival of 11.2 months in KPS $>70^{(21)}$. So according to the above studies and our study also performance status is found to be an important prognostic factor.

Maximal tumor excision is a crucial aspect in the treatment of GBM. In this study 30patients $(68 \%)$ had a complete excision of tumor and 14 patients (32\%) had only a partial excision. The median survival of those with complete excision was 20 months and only partial excision was 10 months ( $\mathrm{p}$ value <0.001) which is statistically significant even though the study population is small. In our study all patients were evaluated with preop and postop MRI scans. This study is a retrospective data based study in our institute and included a small number of patients. The results of the study closely resembled that of the standard studies where survival outcome is improved with gross total excision. This is true even in elderly patients ${ }^{(22)}$. In a study by Jasmine Hager a significant improvement is seen in overall survival (OAS) and progression free survival (PFS) when compared with stereotactic biopsy ${ }^{(22)}$. Straube et al has detected in his study that most patients recur after treatment and the recurrence is most commonly noted within a radius of $2 \mathrm{~cm}^{(15)}$. Study by Clarke et al showed a median PFS after recurrence is 7 months and median OS after recurrence is $22-44$ weeks ${ }^{(23)}$. Elens et al has described that in case of disease recurrence occurring after 6 months repeat gross excision of the tumor has to be considered if feasible (24). Sanai et al analyzed 28 high grade glioma articles and detected that there is evidence that extensive resection was associated with longer life expectancy ${ }^{(25)}$. This is similar to the results obtained by Jalali et al where a median survival of 17 months was seen in patients with complete excision and 6months in patients with partial excision. ${ }^{(26)}$. Metaanalysis conducted by Brown et al of 37 studies with gross total resection the mortality in 1year and 2 years is reduced and so substantially improves the OS and PFS (27). In our study also the median survival is better for patients with complete excision of tumor.

Only those patients who have received the standard treatment protocol of postop concurrent chemoradiation followed by adjuvant temozolomide for 6 months were selected in our analysis, which is the treatment regimen developed after the sentinel study by Stupp et al ${ }^{(9)}$. In our study surgery was followed by a postoperative MRI to assess the extent of resection and the 
residual disease noted to help in planning the adjuvant treatment.

Radiotherapy remains an important treatment modality in brain tumors for a long time to increase the local control, improve quality of life and increase survival. Conventional treatment is delivering of a total dose of $60 \mathrm{~Gy}$ in 30 fractions, 2 Gy per fraction five days per week over a period of 6 weeks with concurrent temozolomide ${ }^{(9)}$, which was the same in our study. Various other radiation doses were investigated by Lawrence et al without an advantage over the above mentioned dose and dose > 60 Gy did not give any advantage over $60 \mathrm{~Gy}{ }^{(28)}$. In GBM involving brain stem lower doses of 54 to $55.8 \mathrm{~Gy}$ in 1.8 Gy per fraction are used ${ }^{(29)}$. In elderly patients short course radiation treatment (40 Gy $/ 15$ fractions) is a reasonable option as it improves the quality of life ${ }^{(29)}$. Reiirradiation is used in younger patients with good performance status even though there is no randomized trial proving a survival advantage ${ }^{(28)}$. Hulshef et al has concluded after analyzing the Dutch Randomized series that hypofractionated radiation represents a radiobiological benefit in poor performance status patients ${ }^{(30)}$.

The standard first line chemotherapy in GBM is temozolomide as a concurrent agent with radiation and as adjuvant chemo drug. The toxicities of the drug are hematological which includes thrombocytopenia, anemia and increase in AST/ALT and nonhematological toxicities like nausea, vomiting, constipation, loss of appetite, anxiety and anorexia ${ }^{(31)}$. The less common side effects are diarrhea, amenorrhea and liver damage ${ }^{(31)}$. In our study the toxicities noticed were fatigue, constipation, nausea and vomiting and these were tolerable. In our study all the patients received concurrent temozolomide and adjuvant temozolomide, as the drug is available to all patients free of cost in our hospital. So comparison between radiation alone versus radiation with temozolomide was not done. In the landmark trial Stupp et al with concurrent chemoradiation the median survival was 14.6 months as against 12.1 months with radiation alone ${ }^{(9)}$. Roldian Urgoiti et al in his study of patients analyzed from the South Alberta Cancer Registry, has looked into the advantage of giving adjuvant temozolomide beyond 6 months and found a median survival of 24.6 months in extended temozolomide and 16.5 months in 6 month treatment ${ }^{(31)}$. Trials have shown that there is no improvement in giving dose dense temozolomide in newly diagnosed patients in spite of the methylation status ${ }^{(31)}$. In our study the prognostic factors detected were age, performance status and extent of resection which is true in other standard trails.

\section{CONCLUSION}

The corner stone of treatment approach in GBM consists of the multimodality treatment by way of maximum debulking, concurrent chemoradiation and adjuvant treatment with temozolomide. This must be combined with improved supportive and palliative care to improve the quality of life and a prolongation in overall survival. Despite these advances in treatment of GBM the 5 year survival remains dismal. So there is indeed a need for improved therapeutic strategies and development of new molecular agents. The prognostic factors which had an influence on median survival were age ( $\mathrm{p}$ value <0.01), performance status ( $p$ value $<0.001)$ and extent of excision of tumor ( $p$ value $<0.001$ ). Novel approaches like immunotherapy, precision chemotherapy and radiotherapy, and targeted therapy which take into consideration the molecular biology of glioblastoma are under investigation and research.

Acknowledgement: None

Conflict of Interest: The authors report no conflicts of interest in the present study.

Source of Funding: None 


\section{REFERENCES}

1. Reis L A, Eismer M P, KosaryC L,Hankey B F et al SEER Cancer statistics 1973-1998 Bethesda M D : National Cancer Institute2001 July;5(2):132-134

2. Ostrum Q T,Gittleman H, Farah P, Ondracek A et al CYBRUS Statistical Report: Primary Brain and CNS tumors diagnosed in the US in 2006-2010 Neuro Oncol 2013:15 Suppl 2:1-56

3. Ghosh M, Subham S, Mandai K, Trivedi V et al. Survival and prognostic factors for Glioblastoma ; Retrospective single institutional study. Indian Journal of Cancer; vol 54,Issue1, January- March 2017:362-7

4. Archya Das Gupta, Tejpal Gupta, Rakesh Jalili ; Indian data on central nervous tumors: A summary of published work , South Asian Journal , 2019 July;5(3):147153.

5. Chamberlain MC. Radiographic patterns of relapse in glioblastoma. Journal of NeuroOncology 2011; 101: 319-323.

6. Engelhard HH, Villano J L, Porter K R, Stewart A K, Baura M, Barker F G et al Clinical presentation, Histology and Treatment in 430 patients with Primary tumors in the spinal cord and spinal meninges or cauda equina $\mathrm{J}$ NeuroSurg Spine 2010:13-67-77

7. Alifiens C, Trafilis D T et al ;Glioblastoma multiforme Pathogenensis and treatment : Pharmacol Ther 2015 Aug 152:63-82

8. Perry J, Zinman L, Chambers A, Spithoff K et al. The use of prophylactic anticonvulsants in patients with brain tumors-A systematic review. Current Oncology 2006;13:222-229

9. Stupp R, Hegi ME, Mason WP, van den Bent MJ et al . Effects of radiotherapy with concomitant and adjuvant temozolomide versus radiotherapy alone on survival in glioblastoma in a randomized phase III study: 5 year analysis of the EORTC-NCIC trial. Lancet Oncol 2009; 10:459-66.

10. Zucchetto M, Catapano C V,, Filippeschi et al: Temozolomide induced differentiation of k562 leukemia cells is not mediated by gene hypomethylation: Current Oncology 2011;14:132-139

11. Stupp R, Mason W P, Vanden Bent M J, Weller et al: Radiotherapy plus concomitant and adjuvant Temozolomide for Glioblastoma: N Eng J Med 2005 March 352(10) 987-96
12. Igor J Barani, David A Larson: Radiation therapy of Glioblastoma :Pub med 2015, PMID 25468225 DOI ;10,1007/978-3-31912048-549

13. Azam F, Latif M F et al Performance Status assessment by using ECOG(Eastern Cooperative Oncology Group) Score for cancer patients by Oncology Health Care Professionals ;Case Rep Oncol 2019;12:728-736.

14. Ohgaki H, Kleihues P (2005) Population based studies on incidence, survival rates and genetic alterations in astrocytic and oligodendroglial gliomas. J Neuropathol Exp Neurol 64:479-489

15. Straube C, Elpula G, Gempt J et al. Reirradiation after gross total resection of recurrent glioblastoma. Strahlen therapie und Onkol.2017;193(11):897-909.

16. Ghosh M, Shubham S, Mandal K, Trivedi V et al, Survival and prognostic factors for glioblastoma multiforme, Retrospective single-institution study; Indian Journal of Cancer,Vol 54;Issue1:January-March 2017

17. Curran WJ Jr, Scott CB, Horton J et al. Recursive Partitioning Analysis of prognostic factors in three Radiation Therapy Oncology Group malignant glioma trials.J Natl CancerInst 1993; 85: 704-10

18. Stupp R, Mason WP, Van den Bent MJ, Weller M, Fischer B et al ; European Organization for Research and Treatment of Cancer Brain Tumor and Radiotherapy Groups : National Institute of Canada Clinical Trial s Group: Radiotherapy plus concommitent and adjuvant temozolomide for glioblastoma .N Eng J Med 2005 352: 987-996

19. Lutterbach J, Sauerbrei W, Guttenberger R et al. Multivariate analysis of prognostic factors in patients with glioblastoma. Strahlenther Onkol; 2003 ; 179:8-15

20. Narendra Kumar, Pankaj Komar, Shabib Lalit Anugana et al. Evaluation of outcome and prognostic factors in patients of glioblastoma multiforme.A single Institution experience, J Neur Sci Rural Pract: 2013 Aug 4;S46-S55. Doi10.4103/09763147.116455

21. Lacroix M, Abi-Said D, Fourney DR et al, A multivariate analysis of 416 patients with glioblastoma multiforme. Prognosis, extent of resection and survival. J Neurosurg 2001;95:190-8 
Bindu S M et.al. Survival and prognostic factors in patients with glioblastoma multiforme receiving radiation and temozolomide - a retrospective study.

22. Jasmin Haher, Euattormann, Sarah Kammerer et al. Impact of resection on overall survival of recurrent glioblastoma in elderly patients; Clinical Neurological and Neurosurgery, vol:174, Nov 2018;19(6):2125

23. Clarke JL, Ennis MM, Yung WKA et al, Is surgery at progression a prognostic marker for improved 6-month progression free survival or overall survival for patients with recurrent glioblastoma? Neuro Oncol. 2011;13(10):1118-1124

24. Elen S, Mandi MD, Dirven MD et al. Repeated surgery for Glioblastoma multiforme : Only in combination with salvage therapy . Surgical Neurology vol69, Issue 5, May 2008, 506-509

25. Sanal N, Polley MY, McDermott MW, Parsa AT et al . An extent of resection threshold for newly diagnosed glioblastomas, J Neurosurg;2011:115-21

26. Jalali R, BasuA, Gupta T, Munshi A, Menon $\mathrm{H}$ et al. Encouraging experience of concommitent temozolomide with radiotherapy followed by adjuvant temozolomide in newly diagnosed glioblastoma multiforme. Single Institution experience. Br J Neurosurg 2007;21:583-7

27. Brown TJ, Brennen MC, Li $\mathrm{M}$ et al. Association of the extent of resection with the survival in glioblastoma; A systematic review and metaanalysis. JAMA Oncol 2016;2:1460-9

28. Lawrence YR, Li XA et al. Radiation dose volume effects in the brain Int $\mathbf{J}$ RadiatOncol Biol Phys.2010:76(3 suppl):S20-S27

29. Kenj Yoshida, Nor Shazrin sulaiman, Daisoka et al, Radiotherapy for Brainstem gliomas in children and adults: A single institution experience and literature review.Asia Pac J Clin Oncol.2017 April;13 (2):e153-e160.doi:10.1111/ajco.12451

30. Hulshof MC, Schimmel EC, Gonzalez D et al. Hypofractionation in glioblastoma multiforme.Radiother Oncol. 2000;54 :1438

31. So Hyun Bae, Min Jung Park, Min Mi Lee et al. Toxicity profile of Temozolomide in the Treatment of 300 Malignant glioma patients in Korea. J Korean Med Science 2014 July ;29(7):980-98

How to cite this article: Bindu S M, P L Rema, Ninan PJ. Survival and prognostic factors in patients with glioblastoma multiforme receiving radiation and temozolomide - a retrospective study. Gal Int J Health Sci Res.2021;6(2):69-78. DOI: https://doi.org/10.52403/gijhsr.20210410 